\title{
Les Indes Orientales Néerlandaises vers 1763-1830. Une pépinière idéale pour une société « en chantier »
}

The Netherlands' East Indies, 1763-1830. A grateful seedbed for engineering society

\section{Angelie Sens}

Traducteur : Pascal Dupuy

\section{CpenEdition} Journals

Édition électronique

URL : https://journals.openedition.org/ahrf/13135

DOI : 10.4000/ahrf.13135

ISSN : 1952-403X

Éditeur :

Armand Colin, Société des études robespierristes

Édition imprimée

Date de publication : 1 mars 2014

Pagination : 161-186

ISBN : 978-2-200-9083-2790-8

ISSN : 0003-4436

Référence électronique

Angelie Sens, «Les Indes Orientales Néerlandaises vers 1763-1830. Une pépinière idéale pour une société " en chantier » », Annales historiques de la Révolution française [En ligne], 375 | janvier-mars 2014, mis en ligne le 01 juillet 2017, consulté le 05 juillet 2021. URL : http://journals.openedition.org/ ahrf/13135; DOl : https://doi.org/10.4000/ahrf.13135 


\title{
LES INDES ORIENTALES NÉERLANDAISES VERS 1763-1830. UNE PÉPINIÈRE IDÉALE POUR UNE SOCIÉTÉ «EN CHANTIER »
}

Angelie SENS

\begin{abstract}
À partir de textes des $\mathrm{XVIII}{ }^{e}$ et $\mathrm{XIX}{ }^{e}$ siècles relatifs à des " projets coloniaux » par des membres néerlandais de l'administration ou des officiers de haut rang des armées coloniales, et à partir du concept de " engineering society » (construction/transformation de la société) l'auteure s'attache, à travers de nombreux exemples pris dans les diverses colonies hollandaises, à saisir les moyens qui étaient alors envisagés afin de renforcer les liens entre la métropole et ses colonies, d'un point de vue politique, économique mais aussi moral. Ces débats, comme le démontre l'auteur, étaient également liés aux discussions concernant le passage d'une économie coloniale d'échanges à de nouveaux modes d'exploitation économique fondés pour la plupart sur le travail libre. Ces projets, habillés d'un discours philosophique et moral issu des Lumières, sont révélateurs de cette période de transition des années 1770 - 1830 dans l'ensemble des métropoles coloniales européennes.
\end{abstract}

Mots-clés : Pays-Bas, colonies hollandaises, engineering society, projets coloniaux, mercantilisme, colonialisme libéral

\section{Introduction}

« Esclave infortuné !-Si ma main, qui ne peut essuyer tes larmes, en fait verser de regret \& de repentir à tes tyrans, je n'ai plus rien à demander aux Indes, j'y ai fait fortune ».

Cette phrase, placée en exergue de la pièce de théâtre de Dirk van Hogendorp, Kraspoekol, of de slaavernij. Een tafereel der zeden van Neerlands Indiën [Kraspoekol, ou l'esclavage. Une histoire morale des 
Indes orientales néerlandaises], 1800, est tirée du Voyage à l'Isle de France $^{1}$ publié anonymement par Bernardin de Saint-Pierre en 1773. Van Hogendorp y plaide pour l'abolition de la traite négrière et de l'esclavage qui, selon lui, ne pouvait advenir que grâce à un renversement moral au sein de la société coloniale ${ }^{2}$.

Dans les Provinces-Unies et leurs colonies, les hommes et les femmes débattaient avec passion de la manière dont il était possible de renforcer les liens entre la métropole et ses colonies, d'un point de vue politique, mais aussi économique et moral. Les concepts de « engineering society » (construction de la société) des $\mathrm{XVIII}^{\mathrm{e}}$ et $\mathrm{XIX}^{\mathrm{e}}$ siècles nous permettent d'éclairer plus finement les idées du temps et la perception des pratiques futures de la société coloniale. Les sources, au cœur de cette contribution, sont constituées de textes des XVIII ${ }^{\mathrm{e}}$ et $\mathrm{XIX}^{\mathrm{e}}$ siècles relatifs à des " projets coloniaux » par des auteurs qui étaient pour la plupart des membres de l'administration ou des officiers de haut rang des armées coloniales et qui avaient, ou prétendaient avoir, une bonne connaissance de tous ces sujets. Et tous, d'une manière ou d'une autre, étaient engagés dans « la construction » de la société coloniale. Leurs écrits reflètent cette période transitoire située entre le « vieil » ordre mercantile et le nouveau colonialisme libéral. Est-il utile de préciser que l'Histoire des Deux Indes de Raynal, les positions d'économie politique d'Adam Smith, de plus en plus répandues grâce au succès international de sa Richesse des Nations (1776), tout comme la philosophie des Physiocrates et des économistes français, donnaient à ces «projets coloniaux » des accents réformateurs, avec une perspective de transformation sociale ${ }^{3}$, d'aspect et de caractère souvent utilitaristes.

(1) Voyage à l'Isle de France, à l'Isle de Bourbon, au Cap de Bonne-Espérance \&c. Avec des observations nouvelles sur la nature \& sur les hommes, par un Officier du Roi, Amsterdam, 1773, 2 tomes. La phrase se trouve à la toute fin du volume II, page 238. « Pour toi, Nègre infortuné qui pleures sur les roches de Maurice, si ma main, qui ne peut essuyer tes larmes, en fait verser de regret \& de repentir à tes tyrans, je n'ai plus rien à demander aux Indes, j’y ai fait fortune ».

(2) Kraspoekol - « frappe dur » - est le nom de la propriétaire d'esclaves de la pièce. L'action se déroule à Batavia (Jakarta), la capitale de Indes orientales néerlandaises. Dans la pièce, Kraspoekol traite ses esclaves de manière très cruelle. Ceci est partiellement dû au fait que les propriétaires femmes d'esclaves à Java sont d'ascendance créole, c'est du moins l'explication qu'en donne Van Hogendorp qui explique par là qu'il leur échappe une partie du caractère civilisé inhérent aux Européens. De nombreux Européens s'adonnent à des pratiques méprisables en raison d'intérêts personnels liés à leur égotisme.

(3) Le terme anglais « engineering » implique toutefois une dimension planifiée et constructiviste que le terme français « transformation » ne rend pas dans son intégralité ; nous l'avons parfois rendu par « construction » ou « fabrique » du social (voir Alain TOURAINE, La production de la société, Paris, Éditions du Seuil, 1973). L'expression «en chantier» nous a toutefois semblé plus appropriée et a été retenue pour la traduction de l'intitulé de l'article (note du traducteur). 
En fait, la majorité des textes relatifs à l'avenir des colonies de l'Empire hollandais ne relèvent pas de la littérature de fiction. En ce qui concerne les genres purement littéraires, que ce soit la poésie, le roman, le théâtre ou la littérature utopique et/ou uchronique, le monde colonial « réel » était souvent décrit par anticipation, et présenté dans un cadre universel où, explicitement ou implicitement, le genre humain et le monde connu étaient, d'une façon ou d'une autre, intégrés.

\section{Quelques remarques historiographiques}

D'un point de vue historiographique, la Compagnie hollandaise des Indes orientales (Vereenigde Oostindische Compagnie, la VOC) a été perçue comme une institution commerciale sans ambition réelle pour la colonisation directe. La VOC s'inscrivait ainsi dans les courants d'échanges asiatiques (ou portugo-asiatiques) déjà existants, notamment celui des épices. Cette vision arbitraire obscurcit la nature bien plus complexe de la VOC et de ses activités aux XVII ${ }^{\mathrm{e}}$ et XVIII ${ }^{\mathrm{e}}$ siècles. L'expansion rapide de ses intérêts commerciaux et territoriaux dans l'hémisphère oriental exigeait d'autres formes d'activité coloniale, mieux adaptées à ces nouvelles situations et bien différentes de simples transactions commerciales traditionnelles. Parmi ces nouvelles situations, on relève des projets de réforme de la société coloniale qui s'accompagnaient simultanément de demandes en hommes et en terres ${ }^{4}$. Mais construire la société coloniale à partir de programmes de colonisation impliquait des transformations qui pouvaient se révéler dangereuses. Des terres cultivables et non fertiles pouvaient « légalement » ou illégalement être obtenues ou simplement confisquées, leurs occupants obligés de changer la nature et le volume de leur production, tout cela aboutissant souvent à des mouvements migratoires « volontaires » ou forcés, relevant tout à la fois de phénomènes attractifs et répulsifs.

Le résultat de la résistance violente contre cette agression coloniale était partie intégrante de la société coloniale. Dans cette mesure, les débats sur la « transition coloniale » globale présumée $(1770-1840)^{5}$ et sur la traite, l'esclavage et son abolition dans le monde de l'Océan indien, sont d'une

(4) Voir par exemple : Rik VAN WELIE, « Patterns of slave trading and slavery in the Dutch colonial world 1596-1863», dans Gert OOSTINDIE (dir.), Dutch colonialism, migration, cultural heritage, Leiden, 2008, p. 155-259, références pages 158-159.

(5) Voir par exemple le numéro spécial de Modern Asian Studies, 38, 3 (2004) : « The Colonial Transition. South Asia, 1780-1840» avec une introduction de Ian J. Barrow et Douglas E. Haynes. 
grande importance ${ }^{6}$. Mais l'historiographie récente a surtout insisté sur « l'identité » et sur les processus de « créolisation » des sociétés coloniales. Comment les sociétés de négoce puis plus tard l'État néerlandais, ont-ils appréhendé des sociétés coloniales dont ils avaient eux-mêmes conçu, de manière d'ailleurs très ordonnée, l'organisation, et qui allaient se révéler des lieux explosifs et traversés par des dynamiques à la fois complexes et singulières ? La formation de nouvelles identités, mais surtout, d'identités mixtes, donna naissance à de nouvelles hiérarchies et à de nouvelles lignes de démarcation entre les groupes sociaux vivant dans les sociétés coloniales, le tout porté par des réseaux familiaux, religieux, de classe ou de race $^{7}$. Les politiques et les activités post-coloniales du $\mathrm{XX}^{\mathrm{e}}$ siècle ont permis l'éclosion de nouvelles réflexions (à partir de l'intégration urbaine) sur les histoires coloniales et impériales récemment regroupées sous l'expression de Nouvelle Histoire Impériale (NHI), un champ à haute dimension culturelle, dans la plus large acception du terme ${ }^{8}$. Tous ces débats peuvent être regroupés à partir du concept du travail (le travail libre, en apprentissage, forcé, migratoire ou encore l'esclavage) et à partir de celui de propriété (propriété territoriale et souveraine, demande de terres par les pouvoirs coloniaux, défrichement ou encore propriété agricole). Ces débats sont liés, d'une manière ou d'une autre, aux discussions qui concernent le passage d'une économie coloniale d'échanges à une économie coloniale fondée sur de nouveaux modes d'exploitation, soutenue par la formation d'États coloniaux par les puissances européennes et sont souvent habillés d'un discours philosophique et moral issu des Lumières.

(6) Voir par exemple Gwyn CAMPBELL (dir.), The structure of slavery in Indian Ocean Africa and Asia, London, 2003 ; Id., (ed.), Abolition and its aftermath in the Indian Ocean, Africa and Asia, London and New York, 2012, et Alessandro STANZIANI (dir.), Labour, coercion, and economic growth in Eurasia, $17^{\text {th }}-20^{\text {th }}$ centuries, Leiden / Boston, 2013. Nous devons mentionner également Anthony REID (dir.), Slavery, bondage \& dependency in Southeast Asia, New York, 1983. Sur un point de vue qui prend en compte les colonies orientales et occidentales, voir : Rik VAN WELIE, « Patterns of slave trading and slavery... », op. cit.

(7) Voir, par exemple, une étude portant sur la colonie du Cap Nigel WORDEN (dir.), Cape Town between East and West. Social identities in a Dutch colonial town, Auckland Park / Hilversum 2012 ; Voir encore un ouvrage récent sur les Indes orientales : Ulbe BOSMA et Remco RABEN, Being "Dutch» in the Indies. A history of creolisation and empire, 1500-1920, Athens, 2008. Enfin, sur les Néerlandais et la côte occidentale de l'Afrique, voir Ineke VAN KESSEL (dir.), Merchants, missionaries and migrants. 300 hundred years of Dutch-Ghanian relations, Amsterdam, 2002.

(8) Le numéro special dirigé par Marieke BLOEMBERGEN et Vincent KUITENBROUWER, « A New Dutch Imperial History » de la revue BMGN-Low Countries Historical Review, vol. 128, nr. 1 (2013) revient sur ce débat historique, né au Royaume Uni, et dans lequel l'histoire des Pays Bas joua un rôle important. Voir en particulier Remco RABEN, « A New Dutch Imperial History ? Perambulations in a prospective world », p. 5-30. 


\section{Débats coloniaux depuis le milieu du XVIII ${ }^{\mathrm{e}}$ siècle}

Comme on le sait, les Provinces-Unies adoptèrent une attitude de neutralité lors de la guerre de Sept ans (1756-63), comme elles le firent d'ailleurs pendant la plus grande partie du XVIII ${ }^{\mathrm{e}}$ siècle. Toutefois, malgré cette neutralité, les conséquences et les résultats de cette guerre se firent sentir dans le pays. Les Bataves furent de plus en plus confrontés à la rivalité avec les Britanniques sur mer et dans leurs colonies orientales, en premier lieu dans leurs comptoirs du sous-continent indien et de l'hémisphère occidental $^{9}$. À partir du milieu du XVIII ${ }^{\mathrm{e}}$ siècle, les Hollandais accélérèrent leur expansion territoriale et renforcèrent leur pouvoir ainsi que leur souveraineté sur Java et son archipel, au détriment des royaumes et des principautés régnantes, selon un processus amorcé au XVII ${ }^{\mathrm{e}}$ siècle, qui devait prendre fin au $\mathrm{XX}^{\mathrm{e}}$ siècle. Tout cela fut mis en place par des traités, par des achats de territoires, par une stratégie qui consistait à diviser pour mieux régner ou tout simplement par la force brutale. En 1749, par exemple, la VOC, basée jusqu'ici en Insulinde, autour de Batavia et dans le Preanger, soit la partie occidentale de Java, réussit à s'agrandir territorialement dans la région du Mataram, au nord-est de l'île, lorsque le Susuhunan (Chef) Pakubuwono II donna sur son lit de mort commandement, pouvoir et autorité (« alle gezag, magt en authoriteyt ») aux Bataves. Autre exemple avec les territoires du Bantam (partie occidentale de Java, à la frontière des possessions bataves en Insulinde) annexés par la force par un gouverneur nommé par les Français, Herman Willem Daendels (18081811), puis sévèrement réorganisés au détriment des princes régnants, comme ce fut le cas avec d'autres territoires en Insulinde. Les Bataves essayaient de trouver d'autres moyens de faire des bénéfices à la fois à partir du commerce international et intra-asiatique et des exportations agricoles ${ }^{10}$. Sur la côte occidentale africaine, les territoires bataves sur la côte de Guinée représentaient une place forte pour le commerce des esclaves, en particulier St George d'Elmina (aujourd'hui au Ghana) tandis qu'au sud se

(9) Dans les colonies orientales, les Néerlandais ont éventuellement perdu leurs comptoirs sur le sous-continent indien et la colonie de Ceylan (en 1796) qui, après les négociations de paix lors du Congrès de Vienne, devaient rester sous emprise britannique. Pour une étude récente sur Ceylan (Sri Lanka), voir Alicia SCHRIKKER, Dutch and British colonial intervention in Sri Lanka c. 1780-1815. Expansion and reform, Leiden, 2006.

(10) Herman Willem DAENDELS, Staat der Nederlandsche Oostindische bezittingen, onder het bestuur van den Gouverneur-Generaal Herman Willem Daendels [...], 's-Gravenhage, 1814, 4 vols., vol. 1, p. 54-61. Voir également F. DE HAAN, Priangan. De Preanger-Regentschappen onder het Nederlandsch Bestuur tot 1811, 4 vols., Batavia / 's-Gravenhage, 1910-1912, vol. 1, p. 474-475. En 1807, Daendels avait été nommé gouverneur par Louis Bonaparte. 
trouvait la colonie du Cap encore plus importante en termes stratégiques et économiques. Il est intéressant de noter que la partie batave de la Guinée faisait partie de la sphère occidentale du trafic commercial transatlantique hollandais tenu par la WIC (West-Indische Compagnie), tandis que la colonie du Cap était contrôlée par la VOC. Au XVII siècle, la place, utilisée comme une simple escale pour les navires se rendant des ProvincesUnies aux Indes du Sud, se transforma en une colonie esclavagiste. Comme ce fut le cas pour Ceylan, les Provinces-Unies devaient perdre la colonie du Cap à la suite du Congrès de Vienne. Dans la République, tout comme dans ses colonies, des initiatives furent engagées afin de remédier aux menaces réelles ou supposées contre l'État, les colonies, la VOC, la WIC, le bien public et la société en général. Pour certains, c'était l'existence même de l'empire colonial qui était en jeu, alors que pour d'autres, il était plutôt question des transformations à venir ${ }^{11}$. En 1778, « l'Oeconomische Tak » (Commissariat économique, ET) fut fondé afin d'endiguer «le déclin progressif de la prospérité hollandaise » et afin d'en augmenter la croissance, le bénéfice étant affecté au service de l'Etat. Le commissariat économique (ET) était une branche de la « Hollandische Maatschappij der Wetenschappen » (Société néérlandaise des Sciences, HMW) basée à Haarlem, une société savante impliquée dans la recherche scientifique théorique et fondamentale.

Le Commissariat, au contraire, présentait explicitement son projet comme relevant d'applications scientifiques. Divisé en sections locales à travers tout le pays et dans les colonies, il connut une augmentation rapide de ses adhésions ${ }^{12}$. En 1778 toujours, la « Bataviaasch Genootschap van Kunsten en Wetenschappen » (la Société Batave pour les Arts et les Sciences, BG) fut fondée à Batavia, alors la capitale des Indes néerlandaises ${ }^{13}$. Malgré ses liens avec de nombreuses sociétés savantes de la République, comme l'HMW et la ET, la Société choisit de rester indépendante, en raison de la distance géographique entre la République et les Indes néerlandaises, mais également en raison de l'importance de la colonisation en Insulinde. Rapidement, on devait retrouver ses membres

(11) Angelie SENS, « Dutch antislavery attitudes in a decline-ridden society, 1750-1815 », dans Gert OOSTINDIE (dir.), Fifty years later. Antislavery, capitalism, and modernity in the Dutch orbit (Leiden, KITLV, 1995), p. 89-104 ; Id., "Mensaap, heiden, slaaf ". Nederlandse visies op de wereld rond 1800, Den Haag, 2001.

(12) J. Bierens DE HAAN, Van Oeconomische Tak tot Nederlandsche Maatschappij voor Nijverheid en Handel, 1777-1952, Haarlem, 1952, p. 1-16.

(13) Pour une récente étude sur les premières 89 années de la BG, voir Hans GROOT, Van Batavia naar Weltevreden. Het Bataviaasch Genootschap van Kunsten en Wetenschappen, 1778-1867, Leiden, 2009. 
dans tous les territoires néerlandais en Asie, mais également sur l'archipel indonésien, le sous-continent indien, au Japon ou encore au Cap. La Société suivait les traces de l'ET en choisissant de donner priorité aux sciences appliquées et pratiques, au savoir utile pour le bénéfice des colonies et de la société coloniale, sans entraver la politique de la VOC et son imposition du secret. Le but ultime recherché par la Société était «d'éclairer » les peuples de l'Est, sans répandre la foi chrétienne ${ }^{14}$; les conversions étaient généralement vues comme des sortes d'échanges culturels, plutôt que relevant du fait religieux. Mais sans l'appui d'inventaires, d'enquêtes, de statistiques et d'éléments cartographiques, la connaissance scientifique dans sa dimension appliquée était extrêmement réduite. C'est la raison pour laquelle, grâce à l'aide de multiples (et internationaux) récits de voyages, ainsi qu'avec l'appui d'autres sources provenant de scientifiques et d'amateurs, de nouvelles investigations et recherches furent entreprises par les membres de la Société, mais également par de simples passionnés, les uns et les autres étant stimulés par des compétitions littéraires ${ }^{15}$ et la promesse de publications ${ }^{16}$. Quant aux sujets qui paraissaient importants aux membres de la Société, ils portaient sur l'agriculture et dans une moindre mesure sur le commerce et le négoce. Ainsi, la flore indigène, tant pour ses vertus médicinales que pour son utilisation économique était un sujet régulièrement traité et débattu.

De même, les recherches agricoles sur les nouveaux modes de culture ou les questions relatives aux cultures commerciales indigènes et exogènes, aussi bien que celles touchant au commerce extérieur, étaient des sujets amplement étudiés par la Société. Mais cette dernière s'intéressait aussi à l'élevage en général et à l'élevage bovin en particulier. On relève des expériences réalisées sur des propriétés appartenant à des membres de la Société ou dans des «jardins » récents. En 1778, la Société souhaita faire l'acquisition d'un jardin près de Batavia afin de s'essayer à la culture de plantes exogènes ${ }^{17}$ et en 1790 on relève l'existence, grâce à la volonté de Frederik Schouwman ${ }^{18}$, membre de la Société, d'un nouveau jardin potager, plus proche de la capitale que le précédent. On

(14) Verhandelingen van het Bataviaasch Genootschap der Konsten en Weetenschappen $(V B G)$, vol. I, Rotterdam, 1781 (1 ${ }^{\text {re }}$ édition Batavia, 1779), p. 3-4.

(15) L'un des premiers membres de la BG fut Willem van Hogendorp. C'est à lui que l'on doit Kraspoekol, of de droevige gevolgen van eene te verre gaande strengheid, jegens de slaaven, Batavia, 1780, qui fut réécrit sous la forme d'une pièce de théâtre, par son fils, Dirk van Hogendorp, vingt ans plus tard.

(16) Verhandelingen van het Bataviaasch Genootschap (Transactions, VBG)

(17) $V B G$, vol. I, Rotterdam, 1781 (1 ${ }^{\text {re }}$ édition Batavia, 1779), p. 39.

(18) Ibidem, vol. V, Batavia, 1790, p. 20. 
note également d'autres thèmes récurrents comme "l'histoire naturelle » conçue de manière très large, puisqu'elle comprenait l'étude de l'homme et de l'humanité. Deux membres de la Société, Josua van Iperen et Jacobus Radermacher, participèrent aux débats scientifiques européens relatifs à l'apparence physique humaine d'un point de vue biologique. Ces débats portaient, entre autres, sur les différences des couleurs de la peau, avec le cas exemplaire de "l'albinos noir $»^{19}$. Enfin, la Société s'engageait dans des recherches portant sur les cultures, les religions, les styles de vie des peuples de l'archipel indonésien, à la fois dans une orientation scientifique, mais également dans l'optique de politiques futures à mener. Pendant ce temps, on relève en 1784, la création de la Société pour le Bien Public [Maatschappij tot Nut van 't Algemeen ('t Nut')]. Comme ce fut le cas avec le ET, la 't Nut' était une organisation divisée en sections locales que l'on retrouvait sur l'ensemble du pays et dans les colonies. Mais la différence essentielle entre les deux sociétés reposait sur le fait que l'ET $s$ 'attachait aux aspects socio-économiques du bien public tandis que la 't Nut' se concentrait sur un programme d'éducation et d'alphabétisation pour tous. En revanche, les deux sociétés se retrouvaient sur la base d'un programme national, voire nationaliste. Dans cette optique, les ProvincesUnies et leurs colonies étaient considérées comme un empire cohérent, formé d'une métropole européenne et d'une wingewesten d'outre-mer, c'està-dire des territoires conquis pour le profit économique et financier (en un modèle centre-périphérie), dont l'économie devait être contrôlée grâce à des mesures protectionnistes. Mais le statut de la VOC était également lié à ces débats. Si des réformes étaient nécessaires, elles ne débouchèrent que sur de nombreuses propositions stériles. Elles relevaient davantage du cataplasme sur une jambe de bois, plutôt que de remèdes fondamentaux, ou même révolutionnaires, auxquels certains aspiraient. Quoi qu'il en soit, la réforme de la VOC devait inévitablement avoir des répercussions sur la société coloniale elle-même. Mais tout cela touchait également aux débats sur la pauvreté et l'assistance publique. Dans ce contexte, l'aide aux pauvres fondée sur des distributions d'amendes et d'autres mesures du même type était considérée comme à trop court terme. Ce dont on avait besoin, c'était une politique économique globale qui stimulerait le secteur agricole aussi

(19) Josua VAN IPEREN, « Beschryvinge van een witte neger van het eiland Bali »,VBG, vol. I, Rotterdam, 1781, p. 307-332 ; Id., « Beschryvinge van een blanke negerin uit de Papoesche eilanden », $V B G$, vol. II, Rotterdam, 1784, p. 229-244 ; Jacobus RADERMACHER, « Proeve nopens de verschillende gedaante en coleur der menschen », VBG, vol. II, Rotterdam, 1784, p. 213-228. Pour ces débats aux Pays Bas, voir Angelie SENS, "Mensaap, heiden, slaaf", op. cit., ch. 2, en particulier p. 55-58. 
bien que le secteur industriel ${ }^{20}$. L'économie coloniale devenait alors un maillon encore plus inoxydable de cette chaîne économique. Après 1815, ce serait la route, encore sinueuse, que les autorités des Provinces-Unies et de leurs colonies allaient suivre.

\section{Projets de colonisation}

La disparition de la VOC, l'éphémère « Paix d'Amiens » et surtout la restitution des anciennes colonies hollandaises en 1815-1816 allaient ouvrir des perspectives jusqu'alors inimaginables. Plans, projets et réformes coloniales pouvaient enfin voir le jour, ou, comme le gouverneur de la colonie du Cap (1803-1806), Willem Janssens, le résuma de manière quelque peu méprisante : l'Europe avait attrapé « la fièvre de la colonisation $»^{21}$. La génération des dirigeants, des décideurs et des «idéologues » qui infiltraient le monde colonial au début du XIX ${ }^{\mathrm{e}}$ siècle provenait en partie des cercles de la VOC, mais ils étaient aussi nombreux à être de nouveaux arrivants sur une scène transformée par la politique du roi Guillaume $\mathrm{I}^{\mathrm{er}}$ et de ses conseillers. Les projets coloniaux étaient fondés idéologiquement sur l'appropriation de la terre et du travail. Le rôle de l'État et de l'exécutif était l'un des facteurs cruciaux de l'expansion territoriale outre-mer, elle-même orchestrée par des individus et des organisations privées ainsi que par l'État lui-même. Toutes ces initiatives formulaient des politiques économiques « libérales » fondées sur un système de sociétés par actions. Ceci explique les propositions du gouvernement néerlandais pour des concessions de propriétés coloniales ainsi que la création « d'associations », à la fois dans les territoires d'outre-mer déjà existants, mais également dans les territoires neufs. Le principe de base reposait sur le droit absolu du propriétaire, grâce au fait que les Européens avaient su créer des lois et des précédents «internationaux $»^{22}$. Les projets coloniaux sont des sources précieuses pour appréhender la vision européenne des peuples africains et asiatiques, qu'ils soient libres ou esclaves. Malgré les préliminaires abolitionnistes dans nombre de ces textes, l'Afrique et l'Asie étaient représentées comme « arriérées », «non-civilisées » et « immatures ». Mais, d'un autre côté, dans la plupart de ces documents, les Africains et les Asiatiques étaient

(20) Voir par exemple J. Bierens DE HAAN, Van Oeconomische Tak tot Nederlandsche Maatschappij, op. cit., 1952, p. 1-37 dans lequel il décrit les idées de Hendrik Herman van den Heuvel, l'un des initiateurs de l'ET, parmi d'autres sujets.

(21) Louis Gerardus Josephus VERBERNE, «Gijsbert Karel en Zuid-Afrika », Historisch Tijdschrift, $14, \mathrm{n}^{\circ} \mathrm{s} 1$ et 2 (1935), p. 5-28 et 143-169, références p. 169.

(22) Voir par exemple Daniel Johannes Pieter ORANJE, Het beleid der Commissie-Generaal. De uitwerking der beginselen van 1815 in het Regeerings Reglement van 1818, Utrecht, 1936, p. 93-95. 
décrits comme potentiellement riches, hospitaliers et travailleurs, à la condition qu'ils acceptent la civilisation européenne et l'indispensable gourou blanc. En revanche, le développement des « croisements de race » comme élément de politique coloniale était de nature récente même si, bien entendu, le processus de « créolisation » était courant dans les sociétés coloniales depuis des siècles. Il était souvent méprisé, voire détesté, et considéré comme une dégénération de la « race blanche ». Toutefois, au XIX ${ }^{\mathrm{e}}$ siècle il devint l'un des moyens proposés pour renforcer l'empire colonial. Mais le plus frappant dans tout cela est le pur optimisme qui transparaît dans cette volonté de réformer et de bâtir un «nouveau monde ». On le perçoit dans des discours idéalistes, dans lesquels subsistent parfois des traces d'utopie, mais qui assurent que ce nouveau monde est à portée de main. Le terme de « civilisation » devient alors un principe structurant de l'universalisme des Lumières. La « Civilisation » était perçue comme un développement progressif menant vers une communauté mondiale « en chantier » et en voie d'achèvement dans un futur proche ${ }^{23}$. Par exemple, Dirk van Hogendorp dans son Proeve over den slavenhandel en de slaavernij, in Neerlands-Indie (Essai sur la traite et l'esclavage aux Indes néerlandaises), le 2 juillet 1796, prédisait qu'il ne faudrait qu'une vingtaine d'années pour préparer la société orientale indienne à une communauté sans esclaves ${ }^{24}$.

De même, en 1816, de leur propre chef, les Gouverneurs «libéraux/progressistes » de l'archipel d'Indonésie, Godert van der Capellen, Leonard Du Bus du Gisignies et Johannes van den Bosch s'embarquèrent dans une mission de civilisation de la société orientale indienne avec la volonté de faire disparaître, sous leur gouvernement, la pauvreté et l'ignorance.

L'un des thèmes était de suivre la libre colonisation européenne en Asie et en Afrique et/ou de la stimuler. Pour certains, la colonisation européenne offrait une réponse partielle aux problèmes socio-économiques intérieurs, comme le chômage, la pauvreté et leurs conséquences financières. Les flux migratoires des Européens vers les colonies outre-mer, concernant à la fois ceux qui se trouvaient dans le besoin mais également ceux possédant un capital à investir, étaient une opportunité permettant de renforcer les économies intérieures et coloniales. Selon certains, une colonie puissante pouvait trancher les liens politiques qui la liaient à la métropole mais,

(23) Angelie SENS, "Mensaap, heiden, slaaf", op. cit., 2001, p. 129-136.

(24) Cet essai était relié à l'ouvrage de Van Hogendorp intitulé Stukken raakende den tegenwoordigen toestand der Bataafsche bezittingen in Oost-Indie en den handel op dezelve, Den Haag and Delft, 1801. 
en devenant un État indépendant, elle pouvait cependant toujours être d'une grande utilité économique pour la métropole et vice versa. Gijsbert Karel van Hogendorp, le jeune frère de Dirk van Hogendorp, partageait cette opinion (avec à l'esprit l'exemple des États-Unis d'Amérique), mais il envisageait un modèle évolutif de partage des biens, plutôt qu'une indépendance révolutionnaire ${ }^{25}$. En 1817, Dirk van Hogendorp publiait son Du système colonial de la France, sous les rapports de la politique et du commerce $^{26}$.

Pourquoi centrait-il son attention sur le cas du système colonial français? Van Hogendorp, qui avait fait sa carrière dans les Indes orientales néerlandaises avait réussi à entrer au service de Napoléon, et il espérait trouver un emploi auprès de l'administration coloniale française dans la mesure où ses chances d'en obtenir un aux Pays-Bas étaient pratiquement nulles, surtout après avoir soutenu l'Empereur lors des Cent-Jours. En 1816, après avoir obtenu sa naturalisation française, il partit, miné par la déception, pour le Brésil ou où il mourut six ans plus tard, alors qu'il était devenu planteur de café. Quoi qu'il en soit, Du système colonial offre à la fois une base théorique mais aussi pratique pour le futur proche de la colonisation française et européenne. Il y distinguait trois types de colonisation : les colonies, les établissements de commerce et les possessions territoriales. Cette distinction était pour lui nécessaire dans la mesure où les futures administrations coloniales allaient être fondées sur le contexte conjoncturel, mais également sur les besoins économiques, politiques et militaires de chaque type de colonisation outre-mer. Mais Van Hogendorp distinguait aussi des sous-genres, comme les colonies pures, les colonies mixtes et les colonies à esclaves. À propos des colonies mixtes, il observait :

«Ce sont celles où la population principale a été transplantée, par la métropole, sur le sol nouveau; mais où elle s'est mélangée avec la population indigène, qui a adopté la religion, les moeurs et les lois du peuple vainqueur, de manière à ne faire qu'une seule et même nation avec lui $»^{27}$.

(25) Gijsbert Karel VAN HOGENDORP, Verhandelingen over den Oost-Indischen handel, Tweede stuk. Behelzende eene staatkundige beschouwing van de kolonien benoorden en beoosten, Kaap de Goede Hoop, met bylagen, Amsterdam, 1802, p. 103, 121-124.

(26) Dirk VAN HoGENDORP, Du système colonial de la France, sous les rapports de la politique et du commerce, Paris, 1817. Voir aussi Jérôme Alexandre SILLEM, Dirk van Hogendorp (1761-1822), Amsterdam, 1890, p. 298-307.

(27) Id., Du système colonial de la France, op. cit., p. 7-9. 
Avec le temps, comme le soulignait Van Hogendorp, de nombreuses colonies européennes étaient devenues des sociétés esclavagistes méprisables. Et comme elles produisaient une faible croissance économique et une prospérité limitée, une nouvelle politique devait être mise en place afin de remplacer l'ancien système colonial. Le but était de trouver de nouvelles « colonies mixtes » (de colonies de plantations) dans les parties fertiles, non encore colonisées, des tropiques. Et de préférence dans des régions peu peuplées où les autochtones organisés sous forme de sociétés tribales, auraient cependant acquis quelques rudiments de civilisation, par exemple par leur attitude envers le travail ou envers la religion chrétienne. Ni la force, ni la violence ne devaient être employées contre eux, mais on devait au contraire faire preuve de persuasion et d'impartialité afin de respecter leurs «droits » naturels. Pour cela on avait besoin d'Européens qui accepteraient volontairement ou par la contrainte (les prisonniers par exemple) d'émigrer outre-mer, s'y installer et se lancer dans les cultures agricoles et industrielles tout en se mélangeant aux populations locales. Et comme les Amériques et les Caraibes étaient déjà sous occupation, Van Hogendorp proposait de se concentrer sur l'Afrique, en particulier sa côte occidentale (le Cap Vert) et à l'Est, l'île de Madagascar, qui pouvaient représenter de nouveaux modèles de colonies françaises ${ }^{28}$.

\section{L'Afrique : Elmina et la colonie du Cap}

Dans le cas des Provinces-Unies, on relève l'élaboration de plusieurs projets d'établissement de nouvelles colonies de plantations en Afrique du Sud et de l'Ouest, autour d'Elmina et à l'intérieur des terres. Au $\mathrm{XVIII}^{\mathrm{e}}$ siècle, les activités dans ce domaine étaient limitées et lorsqu'elles existaient, les initiatives venaient d'organisations privées (la VOC, la WIC) et individuelles. Au début du XIX ${ }^{\mathrm{e}}$ siècle, la colonisation dans cette partie du continent africain était perçue comme une solution à l'abolition à venir de la traite négrière et à ses conséquences futures. Elle était en outre portée par l'État. L'un des arguments développés reposait sur l'idée que sur la terre fertile africaine se trouvait un nombre abondant d'ouvriers agricoles.

(28) Ibidem, p. 78-126. Il est intéressant de relever que les sociétés les plus hautement civilisées de l'Asie ne correspondaient pas au modèle que Van Hogendorp avait en tête. Les besoins portaient sur de petites sociétés dans lesquelles les apprentissages techniques et disciplinaires pourraient se développer sur un terrain fertile. La même chose valait pour les théories sur la conversion des populations telles qu'elles étaient développées chez les missionnaires autour de 1800. Plus développée était une religion, comme l'Islam et l'Hindouisme, plus les conversions étaient difficiles. Voir Angelie SENS, "Mensaap, heiden, slaaf",...op. cit., passim. 
En déplaçant l'économie de plantation des Caraïbes vers l'Afrique, la traite atlantique était vouée à une éventuelle disparition. Entre 1803 et 1806, soit la période pendant laquelle le gouvernement batave reprit possession de la colonie du Cap, le missionnaire Jacobus Kicherer proposa d'établir une bergerie à Grote Zakrivier afin de « civiliser » la région du Khoikhoi, tandis que Gijsbert Karel van Hogendorp présenta un projet d'établissement d'une colonie d'esclaves libres en Plettenbergbaai, peuplée de colons (de préférence des petits fermiers et des artisans), à partir de laquelle les missionnaires civiliseraient et éduqueraient la région du Khoikhoi. Van Hogendorp était un membre distingué de la Société des Missionnaires Hollandais fondée en 1797 (Nederlandsch Zendeling Genootschap, NZG) qui s'était engagée dans un programme de conversion des non-chrétiens dans le monde entier ${ }^{29}$. Van Hogendorp pensait que la colonisation allait augmenter le volume du commerce maritime et dans le même temps alléger la mère-patrie de toute une population au bord de la misère. Il dirigeait son attention vers les «nouvelles » terres au-delà de la ville du Cap.

Les nouveaux arrivants venus des Pays-Bas allaient donner un nouvel élan à la «civilisation européenne $»^{30}$. Selon lui, la réussite reposait sur un système de petites propriétés. Si sa campagne de recrutement d'immigrants et d'investisseurs venus des Pays-Bas sembla un succès ${ }^{31}$, finalement, ce type de projets de colonisation devait rester ce qu'ils étaient au départ : uniquement des projets. Quelques années plus tard, en 1807, le gouverneur d'Elmina, Pieter Linthorst, proposa un projet de colonisation au gouvernement de La Haye.

Après 1814, le ministre néerlandais du Commerce et des Colonies, Johannes Goldberg, essaya de mettre en place ces mêmes projets ainsi que d'autres. Les plantations devaient être établies et exploitées par des Africains (libres). Des jeunes gens européens en parfaite santé, des femmes et des enfants (orphelins de préférence) devaient être incités à émigrer vers l'Afrique. Les mariages mixes seraient admis afin d'élever des enfants pouvant survivre au climat tropical. Outre la création de plantations, il était aussi proposé que le commerce intérieur soit intensifié, tandis que les

(29) Sur les sociétés de missionnaires néerlandaises, voir J. BONESCHANSKER, Het Nederlandsch Zendeling Genootschap in zijn eerste periode. Een studie over opwekking in de Bataafse en Franse tijd, Leeuwarden, 1987 ; Angelie SENS, "Mensaap, heiden, slaaf" op. cit., 2001, ch. 3.

(30) Gijsbert Karel VAN HOGENDORP, Verhandelingen over den Oost-Indischen handel..., op. cit ; Louis Gerardus Josephus VerBERnE, «Gijsbert Karel en Zuid-Afrika », op. cit., n 1, p. 14-19 ; J. BONESCHANSKER, op. cit.... pp. 76-77; Henriette DE BEAUFORT, Gijsbert Karel van Hogendorp. Grondlegger van het Koninkrijk, Rotterdam 1979, p. 186-190. p. $143-144$

(31) Louis Gerardus Josephus VerbERnE, « Gijsbert Karel en Zuid-Afrika », op. cit...n ${ }^{\circ} 2$, 
possibilités d'extraction de l'or seraient étudiées. L'une des pistes évoquées était de faire venir des esclaves instruits des Antilles afin de travailler la terre $^{32}$ en écho aux activités des organisations telles que la Compagnie britannique de la Sierra Leone ou la Société américaine de colonisation, ainsi que leurs projets de colonisation respectifs en Sierra Leone et au Libéria. Herman Willem Daendels arriva à Elmina au début de 1816 comme nouveau gouverneur des « possessions hollandaises sur la côte de Guinée ». Ses instructions étaient vastes et en raison de la mauvaise situation financière du moment, presque impossibles à remplir. En plus d'essayer de mettre fin aux guerres indigènes, de maintenir l'abolition de la traite négrière et de réaliser un inventaire global des établissements coloniaux, il dut également organiser un système de plantation dans lequel la coopération avec les royaumes africains, fournissant terres et travail, devrait être partie intégrante. Ces établissements à venir sur lesquels travaillaient des fermiers africains avaient pour but de produire pour le marché international ${ }^{33}$. La même année, Rogier van Polanen critiqua le programme de colonisation en direction d'Elmina et de ses environs qui était, selon lui, par trop oppressif envers les populations indigènes. Il justifiait en premier lieu ses critiques par la reprise de la traite négrière que cela entraînerait. D'autre part, la tromperie des Européens achetant des terres aux rois et aux princes africains, une fois découverte, entraînerait auprès de ces derniers un sentiment de déception, comme ce fut le cas partout ailleurs. Une augmentation des réclamations de terres conduirait, selon Van Polanen, à une augmentation proportionnelle de la traite négrière (pourtant interdite), accompagnée de terribles conséquences pour les terres intérieures situées en Afrique Occidentale. Les Néerlandais devraient suivre l'exemple de la GrandeBretagne, qui, après l'abolition de la traite négrière, s'était parfaitement maintenue économiquement, compte tenu de l'augmentation du volume des échanges entre l'Afrique et l'Angleterre. Enfin, ajoutait Van Polanen, les Néerlandais devaient aussi s'inspirer de la forte croissance de la colonie libre de la Sierra Leone ${ }^{34}$.

(32) Piet EMMER, Engeland, Nederland, Afrika en de slavenhandel in de negentiende eeuw, Leiden, 1974, Part II, p. 51-82.

(33) Paul VAN 'T VEER, Daendels. Maarschalk van Holland, Zeist / Antwerpen, 1963, p. 199200.

(34) Rogier Gerard VAN POLANEN, Brieven betreffende het bestuur der koloniën en bevattende eene beoordeling van een werkje, over dat onderwerp uitgegeven, getiteld: Java. Waarbij gevoegd zijn eenige belangrijke authentieke stukken, welke een nieuw licht verspreiden over het vorig bestuur aldaar van den Gouverneur-Generaal H.W. Daendels, Amsterdam, 1816, p. 45-50. 
Daendels s'attacha aussi tout particulièrement aux infrastructures. Il fit construire des routes ${ }^{35}$, comme celle entre Elmina et le village de Simbo situé à l'intérieur des terres, où l'eau potable et une main d'oeuvre étaient disponibles. Daendels recruta des cantonniers parmi les Kru (ou Krumen) sur la base d'un salaire mensuel de cinq florins et d'un hébergement gratuit. Son intention était d'utiliser ces Krumen pour travailler dans les plantations ${ }^{36}$.

En février 1817, Daendels indiqua au roi que, selon lui, la Guinée permettrait de remplacer les colonies perdues (Essequebo, Berbice et Demerara, aujourd'hui la Guyana). Il continuait en comparant l'Afrique de l'Ouest à Java : les royaumes africains devaient être réduits à la subordination, comme ce qui fut fait à Java. Mais la plus grande différence à mettre en place entre les deux régions reposait sur le fait qu'en Guinée la propriété foncière légale devrait être fondée sur une base libre et privée. Les propriétaires indigènes devraient exploiter les terres à l'aide d'ouvriers autochtones, soit des esclaves, soit des engagés sous contrat, soit des travailleurs libres. Daendels jugeait nécessaire d'avoir recours au travail des esclaves dans ces plantations africano-européennes mixtes qu'il avait en tête, mais au bout de dix ans de travail, les esclaves devaient être affranchis. Avant tout, il jugeait nécessaire que les familles hollandaises qui possédaient un minimum de ressources financières, migrent vers la côte ouest africaine pour superviser ces colonies de plantation. Il développa même une campagne de recrutement en direction de ces familles aux Pays-Bas ${ }^{37}$.

Après la mort de Daendels en mai 1818 les développements coloniaux connurent un ralentissement. Les projets visant à coloniser des territoires d'Afrique de l' Ouest n'aboutirent pas ou ne furent simplement pas réalisés ${ }^{38}$. À la place d'une colonisation (européenne), le gouvernement néerlandais s'engagea à partir des années 1830 dans un recrutement africain (Ashanti) pour les forces armées de l'archipel indonésien. La guerre de Java (1825-1830) en était la raison immédiate, une guerre qui avait conduit à des pertes militaires (et financières) et à la dévastation des terres agricoles ${ }^{39}$.

(35) L'une des grandes réusssites historiques de Daendels en tant que gouverneur de Java, (ou comme nadirs devrait-on dire), fut le bâtiment de la « Grote Postweg » (Jalan Raya Pos en indonésien), qui a énormément amélioré la diffusion du courrier à Java, mais également le commerce et les transports militaires de l'Ouest vers l'Est.

(36) Paul VAN'T VEER, Daendels. Maarschalk van Holland, op. cit..., p. 201.

(37) Ibidem, p. 201-202.

(38) En 1872 les Néerlandais vendent Elmina aux Britanniques en échange d'Aceh (Sumatra).

(39) Entre 1831 et 1872 plus de 3000 soldats Ashanti furent recrutés pour l'armée royale des Pays-Bas des Indes orientales (Koninklijk Nederlandsch-Indisch Leger, KNIL). Beaucoup de ces 


\section{En Asie : Java}

La colonisation européenne systématique de l'archipel indonésien, notamment de Java, ne fut jamais une priorité des autorités néerlandaises du XVIII ${ }^{\mathrm{e}}$ siècle et des responsables de la VOC. Dans les années 1740 le gouverneur Gustaaf Willem van Imhoff (1743-1750) s'y attacha en essayant en même temps de résoudre un certain nombre de problèmes auxquels la VOC étaient confrontée à cette époque. Il développa ainsi un projet afin d'inciter les petits agriculteurs européens à émigrer vers Java où ils devaient former le noyau d'un début de production agricole européenne et, plus précisément, de production horticole. En tant que fermiers-locataires, ces derniers étaient censés cultiver des légumes, des fruits, ainsi que d'autres cultures en direction de la population urbaine en complément d'une alimentation à base de riz ${ }^{40}$. Van Imhoff s'inspirait de la colonisation européenne de la province du Cap en Afrique du Sud, qui s'était produite sous l'égide de Jan van Riebeeck, à partir du milieu du XVII ${ }^{\mathrm{e}}$ siècle, comme ce serait aussi le cas pour Gijsbert Karel van Hogendorp, quelques soixantes années plus tard ${ }^{41}$. À l'origine une simple étape pour les navires de la VOC, la colonie du Cap devait rapidement devenir une véritable colonie de peuplement, ou une «colonie pure », selon la typologie de Dirk van Hogendorp ${ }^{42}$. Cependant, toutes les possessions d'outre-mer en Afrique et en Asie étaient peuplées par un mélange d'Européens «purs », de «créoles», de populations autochtones ainsi que par des esclaves (importés). Dans les élites coloniales, on pouvait trouver de nombreux descendants de «mariages » mixtes, hommes et femmes mélangés, en une pratique courante. La colonie du Cap ne faisait pas exception à la règle, tout comme Java. Là-bas, les Chinois avaient joué un rôle important en tant que commerçants (sur un plan régional et local) ou comme intermédiaires dans le domaine agricole (plantations et autres), ou encore dans l'industrie et les services ; certains d'entre eux étant même devenus extrêmement riches ${ }^{43}$.

Vers 1800, le « Raad der Asiatische Bezittingen en Etablissementen » (Conseil des possessions et des établissements asiatiques) demanda à des experts, en particulier Dirk van Hogendorp et Sebastiaan Cornelis

hommes étaient des esclaves et des affranchis au moment de leur recrutement. Voir Ineke VAN KESSEL, Zwarte Hollanders. Afrikaanse soldaten in Nederlands-Indië, Amsterdam, 2005. 4, p. 91-129.

(40) J. Bierens DE HAAN, Priangan, op. cit... vol. 1, pp. 266-276, vol. 2, pp. 530-537 et vol.

(41) Gijsbert Karel VAN HOGENDORP, Verhandelingen over den Oost-Indischen handel, op. cit... p. viii-x.

(42) Dirk VAN Hogendorp, Du système colonial de la France, op. cit., p. 8.

(43) Ulbe Bosma et Remco RABEN, Being «Dutch» in the Indies, op. cit..., p. 7-8. 
Nederburgh, de le conseiller sur sa future politique coloniale. L'une des questions qui leur étaient posées était de savoir si, et dans quelle mesure, il serait utile et nécessaire de réformer les possessions des Indes orientales dans la continuité de celles des Antilles. L'autre question était de savoir s'il serait plus rentable pour l'Empire néerlandais et ses habitants de maintenir un système colonial fondé sur une société en situation de monopole ou bien de la remplacer par un système libre-échangiste ouvert à tous les citoyens néerlandais. En cas de réponse affirmative, il fallait aussi en préciser les conditions et les règlements. Dans sa réponse, Nederburgh concluait que la comparaison entre les colonies des Antilles et celles des Indes orientales ne pouvait être établie en raison de leurs caractéristiques très différentes. Les possessions antillaises étaient fondées sur l'esclavage, ce qui n'était pas le cas de celles des Indes orientales. Selon Nederburgh, l'exemple de Saint-Domingue et les conséquences de l'abolition française de l'esclavage était une preuve suffisante que le travail des esclaves sous une forme ou une autre était nécessaire dans ce type de colonies. Java devait être considérée selon ses propres singularités. Les Javanais ne devaient pas être soumis à des changements « révolutionnaires », comme la division et la propriété foncière des terres arables pour le peuple, ou le libre choix du mode de culture, ou encore l'abrogation de la corvée obligatoire, comme Dirk van Hogendorp l'avait proposé. En fin de compte, ce genre de réformes n'entrainerait que misère et esclavage. Le maintien du statu quo, voilà la meilleure chose à faire, concluait Nederburgh ${ }^{44}$.

Dirk van Hogendorp était farouchement opposé au système en vigueur à Java - rien de plus qu'un «système de vol légalisé et de pillage $»-$ et il critiquait Nederburgh pour ses positions conservatrices ${ }^{45}$. Contrairement à Nederburgh, Van Hogendorp se faisait l'avocat du système anglais développé dans le sous-continent indien, et plus particulièrement au Bengale par Lord Cornwallis, depuis les années 1780. Quelques-unes des caractéristiques de ce système reposaient sur les concessions de terres, la

(44) Sebastiaan Cornelis NEDERBURGH, Verhandeling over de vragen: of, en in hoe verre, het nuttig en noodzakelijk zijn zoude, de Oost Indische bezittingen van deezen staat [...] te brengen op den voet der West Indische volkplantingen; [...], Den Haag, 1802, p. 54-65. Il est intéressant de relever que Nederburgh n'insiste pas sur la répartition de la propriété foncière dans les économies de plantations esclavagistes des Antilles.

(45) Ce qu'il fit dans Nadere uitlegging en ontwikkeling van het stelsel van Dirk van Hogendorp. [...]. In antwoord op het onlangs uitgekomen werk van den gewezenen Commissaris Generaal S.C. Nederburgh [...], Den Haag, 1802, p. 1-32. Une année avant qu'il ne publie ses réflexions sur la situation économique et financière des Indes orientales dans Dirk VAN HOGENDORP, Stukken raakende den tegenwoordigen toestand der Bataafsche bezittingen in Oost-Indie en den handel op dezelve, Den Haag and Delft, 1801. 
libre disposition des produits dérivés et un système fiscal équitable. La seule chose que Nederburgh proposait était finalement que le commerçant (le koopman) passe d'une entreprise privée (l'ancienne VOC) à une entreprise d'État (la République batave). Pour Van Hogendorp une telle position était inadmissible ${ }^{46}$.

Sous la gouvernance de Thomas Stamford Raffles (1811-1816) les Européens eurent la possibilité d'acheter ou de louer des terres agricoles dans plusieurs régions de Java, ce qui signifiait une rupture avec les pratiques de la VOC et de ses successeurs. Raffles introduisit également, en 1813-1815, un « système de rente foncière » (landrentestelsel) fondé sur la propriété foncière de l'État colonial, sur la collecte des récoltes et des loyers et sur la mise en location de terres proposées aux fermiers et soumises à des baux de location ${ }^{47}$. Les autorités considéraient comme relevant de leur devoir de protéger les intérêts des autochtones contre les potentiels actes répréhensibles et les atrocités de ces nouveaux propriétaires, mais également contre ceux perpétrés par les Chinois et les Javanais. Concernant ces derniers, il s'agissait de les protéger des agissements des princes ou des hauts fonctionnaires. Mais les citoyens ressentaient aussi une obligation morale de veiller sur les droits, le bien-être et la prospérité de leurs « frères humains ». À la fin de l'année 1815, « La Java Benevolent Institution » fut fondée à Batavia sur le modèle de l'Institut africain britannique ${ }^{48}$ (1807), qui avait lui-même plus ou moins succédé à la Compagnie de la Sierra Leone. L'objectif principal de la Java Benevolent Institution était de contrer la traite des esclaves dans les Indes orientales en aidant au maintien de l'abolition de la traite transatlantique promulguée en 1807-1808, tout en soutenant des solutions alternatives (par le travail) pour les habitants de ces régions afin de faire d'eux des gens civilisés et heureux ${ }^{49}$.

Entre 1816 et 1830, la colonisation européenne dans l'archipel indonésien n'était pas seulement la priorité des responsables politiques néerlandais et indiens, elle provoqua aussi dans les années 1820 un débat politique

(46) Dirk VAN HOGENDORP, Nadere uitlegging en ontwikkeling, op. cit., p. 6-8, 19-21, 33.

(47) Thomas Stamford RAFFLES, The History of Java, 2 vols., Oxford/New York/Melbourne, 1978 (orig. London, 1817), p. 151-161

(48) Sur la naissance de la société philanthropique, voir Java Government Gazette, 9 décembre 1815, p. 3. Le gouverneur Thomas Stamford Raffles était parmi les premiers membres de la société, Java Government Gazette, 9 décembre 1815, p. 4, et 6 janvier 1816. Raffles s'attacha également intensément à ranimer la Société Batave pour les Arts et les Sciences.

(49) L'institution africaine avançait dans ses statuts que ni la colonisation, ni le commerce ne devraient être utilisés afin de « civiliser » l'Afrique et ses habitants, mais « qu'elle devait s'attacher à diffuser le savoir et stimuler l'industrie en Afrique par des méthodes adaptées à la situation et aux coutumes de ses habitants ». Report of the Committee of the African Institution, read to the General Meeting on the 15th July, 1807 [...], London, 1807, p. 55-57, citation p. 56. 
et public enflammé, en particulier lorsque l'on examina attentivement la politique du gouverneur Van der Capellen. Les participants à ce débat évoquaient l'échec de l'expérience de Van Imhoff des années 1740 qui visait à attirer les fermiers européens. Les partisans de la colonisation européenne, le groupe des « réformateurs éclairés », faisaient la promotion d'une colonisation à la solde d'investisseurs européens financièrement solides, qu'ils présentaient comme une solution aux problèmes économiques et financiers auxquels étaient confrontés à la fois la métropole et les Indes orientales.

Après 1816, Européens et Chinois demandèrent l'autorisation du gouvernement de s'implanter comme entrepreneurs agraires ou propriétaires de plantations. Il semblerait que quelques Hollandais se trouvaient parmi eux. Le gouvernement colonial était réticent à répondre favorablement à ces demandes. Il voulait obtenir davantage de temps afin d'étudier les conséquences de la vente et/ou de la location de ces terres. Une telle décision nécessitait l'approbation ou le désaveu du roi Guillaume ${ }^{\text {er }}$. La question traîna pendant de nombreuses années, mais nullement en raison des conséquences prévisibles pour les peuples autochtones pour qui les garanties contre les malversations et l'oppression étaient indispensables ${ }^{50}$.

Le premier gouverneur, après le départ de Java de Raffles, fut G.A.G. $\mathrm{Ph}$. (Godert) van der Capellen (1816-1825/6). En 1815, il partit pour Java en tant que l'un des trois membres de la Commission Générale, avec instruction de récupérer les possessions britanniques des Indes orientales et d'étudier la situation dans laquelle se trouvaient ces colonies. Guillaume Ier adjoint à la Commission, sous le titre de conseiller général, le savant Caspar G. C Reinwardt qui était chargé, quant à lui, de rédiger un rapport de la situation actuelle de Java. Reinwardt envisageait de réformer le système de santé publique ainsi que le système éducatif à Java. Il initia également la création d'un jardin botanique à Buitenzorg, près de Batavia, comme un moyen de favoriser et d'améliorer l'agriculture et enfin s'engagea dans la recherche scientifique dans le domaine de l'« histoire naturelle ». La Commission, accompagnée de Reinwardt, voyagea intensément autour de Java, Sulawesi, aux Moluques et dans les autres îles de l'archipel ${ }^{51}$.

(50) Theo Stevens, Van der Capellen's koloniale ambitie op Java. Economisch beleid in een stagnerende conjunctuur, 1816-1826, Amsterdam, 1982, p. 120-123, 135-140.

(51) Andreas WEBER, Hybrid Ambitions. Science, governance, and empire in the career of Caspar G.C. Reinwardt (1773-1854), Leiden, 2012, p. 115-177 ; sur le jardin botanique, voir p. $132-137$. 
Connu pour son attitude « éclairée » et « libérale », Van der Capellen avait une vision claire des questions socio-économiques. Il proposa d'introduire un système scolaire « moderne », permettant aux enfants autochtones d'apprendre à lire et à écrire. Il déclara que l'éducation devrait être laissée aux mains du gouvernement, et non pas à celles des Églises et des missionnaires. Au lieu de leur enseigner la Bible, ils devaient s'appuyer sur des lectures « éclairantes » afin de devenir d'abord des êtres humains ${ }^{52}$, puis des membres utiles à la société :

«Un meilleur système éducatif doit aller de pair avec la sécurité des biens de la terre, avec l'encouragement à l'assiduité et au travail, avec la protection du gouvernement et avec un traitement équitable et humain de la population dans son ensemble $\gg^{53}$.

À partir de 1815, Johannes van den Bosch publia régulièrement des textes portant sur les colonies d'outre-mer ${ }^{54}$ ainsi que sur les colonies agricoles des Pays-Bas dans une volonté de soulager les pauvres néerlandais, pour lesquels il fonda le «Maatschappij van Weldadigheid 》 (La Société Bénévole) en $1818^{55}$. Dans son étude (1818) en deux volumes sur les colonies situées en Asie, en Amérique et en Afrique, Van den Bosch se penchait sur les échecs des nombreuses expériences qui, tout au long du $\mathrm{XVIII}^{\mathrm{e}}$ siècle et au tout début du XIX ${ }^{\mathrm{e}}$ siècle, avaient cherché à intensifier la production coloniale dans les Indes orientales à destination de l'Europe et/ou du marché mondial. Il distinguait quatre principaux obstacles à la réforme économique et sociale. Tout d'abord, la faible productivité des peuples autochtones, puis le volume insuffisant de la plupart des cultures commerciales, ensuite la «nature » du gouvernement autochtone,

(52) Ido H. ENKLAAR, Joseph Kam. «Apostel der Molukken », 's-Gravenhage, 1963, p. 108, note 58 .

(53) Cité dans Theo STEvens, Van der Capellen's koloniale ambitie op Java, op. cit... p. 3 (traduction de l'auteur)

(54) Anonyme [Johannes VAN DEN BOSCH], Brief, inhoudende eenige onpartijdige aanmerkingen op eene memorie, onlangs in het licht verschenen, onder den titel van: Staat der Nederlandsche Oostindische bezittingen onder het bestuur van den gouverneur-generaal Herman Willem Daendels, 's Gravenhage, 1815. Il fut publié le même jour qui vit le départ de Daendels pour Elmina ; voir J. J. Westendorp BOERMA, Een geestdriftig Nederlander. Johannes van den Bosch, Amsterdam, 1950, p. 18-19 ; Johannes VAN DEN Bosch, Nederlandsche Bezittingen, in Azia, Amerika, en Afrika, in derzelver toestand en aangelegenheid voor dit Rijk, Wijsgeerig, Saatshuishoudkundig en Geographis beschouwd [...], 's Gravenhage et Amsterdam, 1818, 2 vols.

(55) Johannes VAN DEN BOSCH, Verhandeling over de mogelijkheid, de beste wijze van invoering, en de belangrijkste voordelen eener algemeene armeninrigting in het rijk der Nederlanden, door het vestigen eener landbouwende kolonie in deszelfs Noordelijke gedeelte, Amsterdam, 1818. Sur Van den Bosch et «The Benevolent Society », voir J. J. Westendorp BoERMA, Johannes van den Bosch als sociaal hervormer. De Maatschappij van Weldadigheid, Groningen, 1927. 
en termes de travail et de morale, et enfin la puissance inadaptée des Hollandais ${ }^{56}$. Van den Bosch considérait la «nature » du peuple javanais comme l' obstacle principal à une colonisation plus intense d'une île pourtant la plus importante des Indes orientales. La douceur du climat et le sol fertile auraient entraîné le Javanais dans l' " indolence » Associées à un système foncier « féodal » et à des relations de travail singulières, leurs politiques de subsistance étaient responsables de l'attitude des Javanais envers la vie et le travail. Il voyait deux manières de changer cette attitude : l'une était la création d'un marché intérieur pour des biens de consommation (européens) et l'autre était de les forcer à travailler par la perception de taxes ou d'impôts en nature. Ce n'était, selon lui, que par un système de travail forcé qu'il était possible de générer des revenus suffisants pour maintenir une colonie viable et saine. Et s'il reconnaissait que les Européens payaient très mal les Javanais pour le fruit de leur labeur, ce salaire devait être considéré comme un simple encouragement afin de les former à devenir de véritables ouvriers agricoles disciplinés. Ce n'était pas un véritable salaire, la rétribution d'un travail fourni. Les gouvernements néerlandais et coloniaux devaient donc s'attacher à rendre le système de travail forcé moins désagréable ou encore lui donner l'apparence d'un régime fiscal équitable. Afin d'éviter que le peuple pense qu'il était forcé de payer des impôts pour les Hollandais - les Javanais n'avaient aucun sentiment d'appartenance à la Hollande, remarquait, avec un grand sens de la litote, Van den Bosch - il était nécessaire de les lever par l'intermédiaire des souverains et des gouverneurs indigènes et ainsi en conserver le décorum. Les deux seules façons de rendre les Javanais imposables étaient soit par la pure violence, soit par l'usage de la ferme autorité des principautés indigènes théoriquement indépendantes. La violence et la guerre s'étaient révélées être pires que le mal, de telle sorte que la deuxième option était la meilleure façon de sécuriser les possessions coloniales et les revenus qu'elles généraient au profit de la métropole ${ }^{57}$.

Toutefois, un changement fondamental avait eu lieu dans la position au sein de la société des élites indigènes. En effet, «l'ancien régime » reposait sur un modèle de gouvernement indirect, mais les réformes administratives de Daendels l'avaient remplacé par un modèle de règlement direct qui incorporait les élites indigènes dans une bureaucratie néerlandoeuropéenne, ce qui avait eu pour résultat de les dépouiller de leur autorité

(56) Johannes VAN DEN BosCH, Nederlandsche Bezittingen, in Azia, Amerika, en Afrika [...], op. cit..., vol. 1, p. 217-253.

(57) Id., Brief, inhoudende eenige onpartijdige aanmerkingen, 1815, p. 3-4, 7-16, 78-79. 
« traditionnelle ». Van den Bosch préférait le modèle indirect car le nouveau système administratif avait conduit à des troubles, à des conflits endogènes et même à la guerre. La guerre de Java (1825-1830) en était un excellent exemple, comme il devait l'expliquer dans les années $1830^{58}$. Alors que Dirk van Hogendorp fondait principalement ses réformes sur la terre (sur la propriété et le libre choix de la culture), Van den Bosch, quant à lui, s'attachait avant tout au travail (sa disponibilité et son usage), son raisonnement reposant sur le développement éducatif et économique du travail. En autodidacte, Van den Bosch étudiait l' «économie politique »de son temps, en s'appuyant sur les travaux d'Adam Smith ainsi que sur ceux d'autres penseurs influents. Mais son expérience personnelle aux Indes orientales (il fut en poste à Java entre 1800 et 1810 avant d'être expulsé par le gouverneur Daendels) lui avait fourni une connaissance pratique et la compréhension de la gestion des plantations coloniales et des problèmes qui en découlaient. La recherche de solutions aux obstacles culturels, sociaux et même psychologiques du développement d'une force de travail organisée et disciplinée dans les colonies et même dans les Pays-Bas, l'amena à combiner les concepts économiques et politiques à l'économie sociale et morale. Il fut capable de mettre en pratique ses politiques et activités « constructivistes » en métropole et dans les colonies en tant que gouverneur à la fois des colonies antillaises et des Indes orientales et comme ministre des Colonies sous Guillaume Ier ${ }^{59}$.

Une des stratégies pour résoudre les problèmes politiques et économiques qui frappaient à la fois la métropole et les colonies fut la création de la « Nederlandsche Handel Maatschappij » (« Compagnie Néerlandaise de Commerce » NHM) en 1824. La NHM était une société royale active dans plusieurs secteurs : commerce, banque, manufacture et prévoyance sociale. Elle lança un programme fondé sur l' « économie sociale » plutôt que sur la nouvelle science libérale de l'économie politique. Certains considéraient le NHM comme le successeur « moderne » de la VOC, mais elle représentait en fait un phénomène tout à fait nouveau. Le principe de la NHM consistait

(58) Albert Schrauwers, «The "Benevolent" Colonies of Johannes van den Bosch. Continuities in the administration of poverty in the Netherlands and Indonesia », Comparative Studies in Society and History, vol. 43, $\mathrm{n}^{\circ} 2$ (2001), p. 298-328, p. 318-319. Vingt ans après sa mort, les réflexions de Johannes van den Bosch furent publiées : Mijne verrigtingen in Indië. Verslag van $Z$. Excellentie den Commissaris Generaal J. van den Bosch, over de jaren 1830, 1831, 1832 en 1833, door Z. Excell. zelv' opgesteld en overhandigd aan zijnen opvolger den Gouverneur Generaal ad interim J.C. Baud [...], Amsterdam, 1864.

(59) Une très intéressante comparaison entre les politiques de Van den Bosch - sur la pauvreté et le secours aux pauvres - dans les Pays-Bas et ses colonies est proposée par Albert SCHRAUWERS, «The "Benevolent" Colonies of Johannes van den Bosch », op. cit., p. 298-328. 
à gérer « une série de filières interconnectées » auxquelles les Néerlandais ainsi que les sujets des colonies (de Java) étaient reliés et qui desservaient les (nouveaux) marchés intérieurs à la fois aux Pays-Bas et outre-mer ${ }^{60}$. Mais l'administration de Van der Capellen fut accueillie par de sévères critiques tant à Java qu'en métropole. À son départ de Java en 1826, les Indes orientales étaient dans un état de quasi-faillite. L'un de ses plus virulents détracteurs était Jacobus Elisa Doornik. Ce médecin et chirurgien, qui avait quitté les Pays-Bas en 1817 pour émigrer à Java, publia à la fin de 1826 son Vrijmoedige Gedachten over Neêrlands Indië (Pensées franches sur les Indes orientales néerlandaises) dans lequel il accusait Van der Capellen et son administration de s'être engagés dans des politiques contraires aux ordres de Guillaume $\mathrm{I}^{\mathrm{er}}$. Même si ces ordres étaient pour le moins vagues et imprécis, leur fondement reposait sur un socle libéral, établi sur le libre-échange, le libre choix des cultures, l'abolition de l'esclavage et du travail forcé, l'autonomie des colons et, enfin, un nombre le plus limité possible de fonctionnaires. La colonisation européenne devrait être encouragée par l'octroi de terres, en favorisant l'immigration des Européens vers l'Est. Mais, en 1823, Van der Capellen devait suspendre la vente et/ou la location de terres, ce qui aboutit à des débats enflammés et à des lettres de mécontentement envoyées à Guillaume $\mathrm{I}^{\mathrm{er}}$.

Tout comme Gijsbert Karel van Hogendorp l'avait proposé pour la colonie du Cap une vingtaine d'années auparavant (avec à l'esprit l'exemple des États-Unis), Doornik opta pour une position beaucoup plus indépendante de l'archipel indonésien vis-à-vis de la métropole, parce que « l'indépendance est un principe inné de chaque colonie ». Sur le long terme, précisait Doornik ${ }^{61}$, toutes les colonies devaient devenir indépendantes afin d'être utiles et rentables pour l'Europe. Mais Vrijmoedige Gedachten fut considéré comme un danger pour l'État et Doornik dut s'enfuir pour les États-Unis ${ }^{62}$.

(60) Albert SCHRAUWERS, «Policing production. Corporate governmentality and the Cultivation System », Focaal. Journal of Global and Historical Anthropology, $\mathrm{n}^{\circ} 61$ (2011), p. 75-90, en particulier p. $76,79-80$.

(61) Jacobus Elisa DoORNIK, Vrijmoedige gedachten over Neêrlands Indië, Amsterdam, 1826, p. 30. Doornik consacra un chapitre à la colonisation, p. 160-219.

(62) Wim VAN DEN DOEL et Pierre VINKEN, « Jacob Elisa Doornik. Een vroeg-negentiendeeeuws koloniaal criticus », Tirade et 47 (2003), p. 77-99. Certains qualifient même Doornik de subversif. En 1808 il publia Wijsgeerig-natuurkundig onderzoek aangaande den oorspronglijken mensch, en de oorspronglijke stammen van deszelfs geslacht, dans lequel il établissait la provenance de l'humanité des anthropoïdes et développait des idées monogéniques et polygéniques, soit des sujets considérées comme une atteinte sévère à la foi chrétienne et à la Bible. Voir Angelie SENS, "Mensaap, heiden, slaaf" op. cit., 2001, p. 48-49, p. 59-60. 
Le nouveau gouverneur, Leonard Du Bus de Gisignies, présenta en 1827 son « Rapport de colonisation » dans lequel une fois pour toutes, il essayait de résoudre plusieurs questions (encombrantes). Il proposait de permettre et d'encourager les investissements privés des Néerlandais ainsi que ceux des «étrangers ». Mais le commerce de produits coloniaux devait rester entre les mains des Néerlandais, soit des entrepreneurs privés, soit de l'État (ou d'une organisation telle que la NHM). Du Bus de Gisignies, assisté de Willem van Hogendorp (le fils aîné de Gijsbert Karel), s’inspira du « système libéral » de Dirk van Hogendorp et du « système de la rente foncière » de Raffles. Mais, selon lui, le «système de la rente foncière » ne conduisait pas à une juste redistribution des terres, seule l'extension des terres arables le permettrait. De là, il aboutit à une proposition de vente de terres incultes, mais fertiles, aux «capitalistes européens »: des investisseurs privés et de futurs planteurs. Selon Du Bus de Gisignies, la sous-production des cultures commerciales provenait de l'insuffisance des investissements en capital et non en raison du maintien d'une véritable société « féodale » javanaise oppressive ${ }^{63}$. Nous avons mentionné un peu plus haut le successeur de Du Bus, Johannes van den Bosch, qui devait obtenir une reconnaissance internationale avec sa Benevolent Institution aux Pays-Bas et l'introduction de la « Cultuurstelsel » (système de culture) à Java, qui allait fonctionner de 1830 à 1870. Mais il s'agit là d'expériences portant sur des périodes chronologiques situées au-delà de cet article ${ }^{64}$.

Certes, les Indes orientales néerlandaises ont été considérées comme une pépinière idéale de projets pour une société en «chantier», mais seulement lorsque les conditions du début du $\mathrm{XIX}^{\mathrm{e}}$ siècle pour la mise en œuvre de ces projets de colonisation ont semblé être réunies, parfois même uniquement dans l'esprit de leurs concepteurs. Au cours du XVIII ${ }^{\mathrm{e}}$ siècle, l'accent a été souvent placé sur la recherche de moyens internes afin de guérir un corps malade - qu'il s'agisse de la VOC (et de la WIC) ou des sociétés néerlandaises et coloniales au sens large. Dans ce cadre, des sociétés sans lendemains furent fondées et elles agirent comme des

(63) «Rapport van den Commissaris-Generaal Du Bus, over het stelsel van kolonisatie » [1 May1827 ; avec des annexes] : (re)publié dans D. C. STEIJN PARVÉ (samenst.), Het koloniaal monopoliestelsel getoetst aan geschiedenis en staatshuishoudkunde, Zaltbommel, 1851; Bart DE PRINS, Voor keizer en koning. Leonard Du Bus de Gisignies 1780-1849. Commissaris-Generaal van Nederlands-Indië, Amsterdam, 2002, p. 153-177.

(64) Sur Van den Bosch et les systèmes de cultures, voir par exemple Albert SCHRAUWERS, «Policing production », op. cit... et Ulbe BosmA, « Dutch imperial anxieties about free labour, penal sanctions and the right to strike », dans Alessandro STANZIANI (dir.), «Labour, Coercion » op. cit...., p. 63-85. 
intermédiaires et des plates-formes pour des débats portant sur la dimension pratique des réformes et des redressements envisagés. Autour de 1800, et surtout après 1816 , se sont présentées des circonstances favorables à la mise en place de réformes et de changements plus radicaux, sollicités par l'État naissant - et centralisé - de Guillaume I ${ }^{\text {er }}$. La nouvelle génération de conseillers, de dirigeants et d'hommes d'État - dans les colonies dont beaucoup avaient fait carrière dans la métropole ou dans les colonies pendant les années révolutionnaires et/ou sous la domination française, a joué un rôle déterminant dans cette histoire. Leurs discours, fondés sur les Lumières, sur le « libéralisme » et l'abolition de la traite négrière et de l'esclavage, ont obtenu une audience de plus en plus large auprès d'un public lui-même de plus en plus réceptif. Ancrés dans les nouveaux concepts de « civilisation », ces discours fondés sur des idées essentialistes donnèrent naissance aux idéaux de constructivisme social.

$\mathrm{Au}$ début du $\mathrm{XIX}^{\mathrm{e}}$ siècle, on peut avancer l'idée que les notions d'interdépendance et de réciprocité entre les Pays-Bas et leurs colonies étaient définitivement nouvelles, les activités de Johannes van den Bosch et l'établissement de la NHM en étant des exemples éloquents. Souvent présentées en termes d'économie et d'économie politique, ces politiques et stratégies nouvelles englobaient pourtant un champ conceptuel beaucoup large. Elles renvoyaient aussi à la politique sociale, à la protection sociale ainsi qu'à des politiques culturelles, scientifiques et éducatives. Mais la métropole n'était pas considérée sur le même plan que ses colonies. Comme c'était déjà le cas au XVIII ${ }^{\mathrm{e}}$ siècle, les colonies dans leur ensemble étaient perçues comme des «wingewesten» exploitées au profit de la métropole. Les projets de colonisation des trois premières décennies du $\mathrm{XIX}^{\mathrm{e}}$ siècle furent souvent présentés avec optimisme et avec une foi inébranlable dans le succès final de cette société en «chantier ». Mais les succès étaient tributaires des capitaux et des investissements humains fournis par des organismes privés et par un État solide, par la mise en place d'infrastructures matérielles et mentales dans les colonies, et par la volonté des peuples autochtones eux-mêmes d'accepter une sorte de culture occidentale (religion, philosophie du travail) sous la douce et sensible direction des Européens. Les projets élaborés pour les colonies africaines furent de courte durée : la colonie du Cap fut perdue au profit des Britanniques et l'intérêt pour Elmina fut bien trop faible. En revanche, les projets à destination de l'archipel indonésien, en particulier de Java, semblaient beaucoup plus prometteurs. Mais autour de 1830, l'optimisme initial d'une société coloniale « construite » sur la persuasion, le respect des contrats équitables et des populations autochtones, s'évanouit. La guerre de 
Java mit en lumière la faiblesse de l'État (financière et militaire) et prouva que la volonté des populations autochtones de suivre volontairement la domination coloniale restait une vue de l'esprit. Les politiques de Van der Capellen, la création de la NHM et, finalement, la mise en place d'un système de culture forcée devaient conduire à l'émergence d'un « industrialisme agraire $»^{65}$.

Angelie SENS

senior researcher

International Institute of Social History Amsterdam angelie.sens@iisg.nl asens@xs4all.nl Traduction assurée par Pascal Dupuy 